Fluctuation and Noise Letters

Vol. 5, No. 0 (2005) 000-000

(c) World Scientific Publishing Company

\title{
GENERALIZED WIENER PROCESS AND KOLMOGOROV'S EQUATION FOR DIFFUSION INDUCED BY NON-GAUSSIAN NOISE SOURCE
}

\author{
ALEXANDER DUBKOV \\ Radiophysics Department, Nizhny Novgorod State University, 23 Gagarin Ave., \\ Nizhny Novgorod, 603950 Russia \\ dubkov@rf.unn.ru \\ BERNARDO SPAGNOLO \\ INFM and Dipartimento di Fisica e Tecnologie Relative, Group of Interdisciplinary Physics, \\ Università di Palermo, Viale delle Science pad. 18, I-90128 Palermo, Italy \\ spagnolo@unipa.it \\ Received (received date) \\ Revised (revised date) \\ Accepted (accepted date)
}

\begin{abstract}
We show that the increments of generalized Wiener process, useful to describe nonGaussian white noise sources, have the properties of infinitely divisible random processes. Using functional approach and the new correlation formula for non-Gaussian white noise we derive directly from Langevin equation, with such a random source, the Kolmogorov's equation for Markovian non-Gaussian process. From this equation we obtain the FokkerPlanck equation for nonlinear system driven by white Gaussian noise, the KolmogorovFeller equation for discontinuous Markovian processes, and the fractional Fokker-Planck equation for anomalous diffusion. The stationary probability distributions for some simple cases of anomalous diffusion are derived.
\end{abstract}

Keywords: Non-Gaussian white noise, Infinitely divisible distribution, Kolmogorov's equation, Wiener process.

\section{Introduction}

Stochastic dynamics is useful to model many biological, chemical, economical and physical systems. The random driving forces have very different origins, in most cases they are Gaussian white or colored noise sources, but often these forces must be considered as non-Gaussian ones, for example, in sensory and biological systems [1]. Moreover, in many physical and biological systems a deviation of real statistics of fluctuations from Gaussian law, leading to anomalous diffusion, is observed [2,3]. A suitable mathematical model to describe the anomalous diffusion is the fractional Fokker-Planck equation, which can be derived from different theoretical approaches 
Generalized Wiener process and Kolmogorov's equation...

[3-7].

In this paper we show that the increments of generalized Wiener process, representing the integral of non-Gaussian white noise, have the properties of infinitely divisible random processes. This enables us to obtain the characteristic functional of non-Gaussian white noise. Then, by using functional approach to split the correlation between stochastic functionals, we derive the Kolmogorov's equation directly from Langevin equation with non-Gaussian white noise source. From this general equation we can obtain the Fokker-Planck equation for continuous Markovian processes, the Kolmogorov-Feller equation for discontinuous Markovian processes and the fractional Fokker-Planck equation for anomalous diffusion.

\section{Generalized Wiener process}

It is well known that Wiener process $\eta(t)$ has stationary and independent increments, and its time derivative $\xi(t)=\dot{\eta}(t)$ is Gaussian white noise. The correlation function of the increments $\eta(t+T)-\eta(t)$ depends on time difference $T$ only, and increments of non-overlapping time intervals are statistically independent. Let us consider now a non-Gaussian random process with stationary and independent increments which can be called as generalized Wiener process. We can represent the increment $\eta_{T} \equiv \eta(T)-\eta(0)$ as

$$
\eta_{T}=\sum_{k=1}^{n}[\eta(k \Delta t)-\eta((k-1) \Delta t)],
$$

where $\Delta t=T / n$. Since the random variable $\eta_{T}$ can be divided into the sum of arbitrary number of independent and identically distributed random variables, its probability distribution belongs to the class of infinitely divisible distributions. Hence, we can express the logarithm of characteristic function of the random value $\eta_{T}$ in the Lévy-Khinchine form [8]

$$
\phi_{T}(u) \equiv \ln \theta_{T}(u)=\ln \left\langle e^{i u \eta_{T}}\right\rangle=\int_{-\infty}^{\infty} \frac{e^{i u x}-1-i u \sin x}{x^{2}} M_{T}\{d x\},
$$

where $M_{T}\{d x\}$ is the canonical measure which, according to definition, takes positive values on a finite intervals, and the integrals

$$
M_{T}^{+}(x)=\int_{x}^{\infty} \frac{M_{T}\{d y\}}{y^{2}}, \quad M_{T}^{-}(-x)=\int_{-\infty}^{-x} \frac{M_{T}\{d y\}}{y^{2}}
$$

converge for any positive $x$ and $T$. Introducing the non-negative density $\rho_{T}(x)$ of the canonical measure: $M_{T}\{d x\}=\rho_{T}(x) d x$, defined in generalized sense, we can rewrite Eq. (2) as

$$
\phi_{T}(u)=\int_{-\infty}^{\infty} \frac{e^{i u x}-1-i u \sin x}{x^{2}} \rho_{T}(x) d x
$$

For two consecutive time intervals $T$ and $S$, we have

$$
\theta_{T+S}(u)=\left\langle e^{i u \eta_{T+S}}\right\rangle=\left\langle e^{i u\left(\eta_{T}+\eta_{S}\right)}\right\rangle=\left\langle e^{i u \eta_{T}}\right\rangle\left\langle e^{i u \eta_{S}}\right\rangle=\theta_{T}(u) \theta_{S}(u),
$$


and

$$
\phi_{T+S}(u)=\phi_{T}(u)+\phi_{S}(u) .
$$

Because $u$ is an arbitrary parameter, we obtain from Eqs. (3) and (4)

$$
\rho_{T+S}(x)=\rho_{T}(x)+\rho_{S}(x), \quad \forall x .
$$

The differentiable solution of Eq. (5), regarding parameter $T$, is only linear one

$$
\rho_{T}(x)=T \rho(x) \text {. }
$$

So, from Eq. (3) we obtain

$$
\phi_{T}(u)=T \int_{-\infty}^{\infty} \frac{e^{i u x}-1-i u \sin x}{x^{2}} \rho(x) d x,
$$

where the kernel $\rho(x) \geq 0$.

\section{Functional correlation formula for non-Gaussian white noise}

The time derivative of generalized Wiener process $\xi(t)=\dot{\eta}(t)$ is a stationary random process and has analogy to Gaussian white noise. Now we derive the characteristic functional of this non-Gaussian delta-correlated noise. By definition, we have

$$
\begin{aligned}
\Theta_{t}[u] & =\left\langle\exp \left\{i \int_{0}^{t} \xi(\tau) u(\tau) d \tau\right\}\right\rangle=\left\langle\exp \left\{i \int_{0}^{t} u(\tau) d \eta(\tau)\right\}\right\rangle \\
& =\left\langle\exp \left\{i \lim _{\delta_{\tau} \rightarrow 0} \sum_{k=1}^{n} u\left(\vartheta_{k}\right)\left[\eta\left(\tau_{k}\right)-\eta\left(\tau_{k-1}\right)\right]\right\}\right\rangle \\
& =\lim _{\delta_{\tau} \rightarrow 0}\left\langle\prod_{k=1}^{n} \exp \left\{i u\left(\vartheta_{k}\right)\left[\eta\left(\tau_{k}\right)-\eta\left(\tau_{k-1}\right)\right]\right\}\right\rangle=\lim _{\delta_{\tau} \rightarrow 0} \prod_{k=1}^{n} \theta_{\Delta \tau_{k}}\left(u\left(\vartheta_{k}\right)\right)
\end{aligned}
$$

where $\vartheta_{k}$ is some internal point of time interval $\left(\tau_{k-1}, \tau_{k}\right), \delta_{\tau}=\max _{k} \Delta \tau_{k}, \Delta \tau_{k}=$ $\tau_{k}-\tau_{k-1}\left(\tau_{0}=0, \tau_{n}=t\right)$, and $\theta_{\Delta \tau_{k}}$ is the characteristic function of increments. To obtain Eq. (7) we used the statistical independence of increments of generalized Wiener process $\eta(t)$. Further from Eqs. (6) and (7) we obtain

$$
\begin{aligned}
\Theta_{t}[u] & =\lim _{\delta_{\tau} \rightarrow 0} \prod_{k=1}^{n} \exp \left\{\Delta \tau_{k} \int_{-\infty}^{\infty} \frac{e^{i u\left(\vartheta_{k}\right) x}-1-i u\left(\vartheta_{k}\right) \sin x}{x^{2}} \rho(x) d x\right\} \\
& =\exp \left\{\lim _{\delta_{\tau} \rightarrow 0} \sum_{k=1}^{n} \Delta \tau_{k} \int_{-\infty}^{\infty} \frac{e^{i u\left(\vartheta_{k}\right) x}-1-i u\left(\vartheta_{k}\right) \sin x}{x^{2}} \rho(x) d x\right\} \\
& =\exp \left\{\int_{0}^{t} d \tau \int_{-\infty}^{\infty} \frac{e^{i u(\tau) x}-1-i u(\tau) \sin x}{x^{2}} \rho(x) d x\right\}
\end{aligned}
$$

Now we derive a useful correlation formula for stochastic functionals of non-Gaussian white noise. We use the generalization of Furutsu-Novikov formula [9], to split the 
Generalized Wiener process and Kolmogorov's equation...

correlation between a stochastic functional $R_{t}[\xi]$ of non-Gaussian random process $\xi(\tau)$, defined on the observation interval $\tau \in(0, t)$, and the process $\xi(t)$ itself

$$
\left\langle\xi(t) R_{t}[\xi+z]\right\rangle=\left.\frac{\dot{\Phi}_{t}[u]}{i u(t)}\right|_{u=\frac{\delta}{i \delta z}}\left\langle R_{t}[\xi+z]\right\rangle .
$$

Here $z(t)$ is an arbitrary deterministic function, and $\Phi_{t}[u]=\ln \Theta_{t}[u]$. From Eq. (8) we obtain the following expression for variational operator in Eq. (9)

$$
\frac{\dot{\Phi}_{t}[u]}{i u(t)}=\int_{-\infty}^{\infty} \frac{e^{i u(t) x}-1-i u(t) \sin x}{i u(t) x^{2}} \rho(x) d x=\int_{-\infty}^{\infty} \frac{\rho(x)}{x^{2}} d x \int_{0}^{x}\left[e^{i u(t) y}-\cos y\right] d y .
$$

Substituting this expression in Eq. (9) we arrive at

$$
\left\langle\xi(t) R_{t}[\xi+z]\right\rangle=\int_{-\infty}^{\infty} \frac{\rho(x)}{x^{2}} d x \int_{0}^{x}\left[\exp \left\{y \frac{\delta}{\delta z(t)}\right\}-\cos y\right]\left\langle R_{t}[\xi+z]\right\rangle d y .
$$

By inserting the operator of functional differentiation into the average and by putting $z=0$, we get finally

$$
\left\langle\xi(t) R_{t}[\xi]\right\rangle=\int_{-\infty}^{\infty} \frac{\rho(x)}{x^{2}} d x \int_{0}^{x}\left[\left\langle\exp \left\{y \frac{\delta}{\delta \xi(t)}\right\} R_{t}[\xi]\right\rangle-\left\langle R_{t}[\xi]\right\rangle \cos y\right] d y .
$$

\section{Derivation of Kolmogorov's equation}

Let us consider now the Langevin equation with a non-Gaussian white noise $\xi(t)$

$$
\dot{x}=f(x, t)+g(x, t) \xi(t) .
$$

By differentiating with respect to time the following expression for probability density of the random process $x(t)$

$$
W(x, t)=\langle\delta(x-x(t))\rangle,
$$

and taking into account Eq. (11), we obtain

$$
\frac{\partial W}{\partial t}=-\frac{\partial}{\partial x}[f(x, t) W]-\frac{\partial}{\partial x} g(x, t)\langle\xi(t) \delta(x-x(t))\rangle .
$$

By using functional differentiation rules and following the same procedure of Ref. [10], from Eq. (11) we get

$$
\frac{\delta}{\delta \xi(t)} \delta(x-x(t))=-\frac{\partial}{\partial x} g(x, t) \delta(x-x(t)) .
$$

Thus, the operator $\delta / \delta \xi(t)$ of functional differentiation with respect to the function $\delta(x-x(t))$ is equivalent to the ordinary differential operator $-\partial / \partial x(g(x, t))$. Using Eq. (10) in Eq. (13) and taking into account Eq. (14), we obtain the Kolmogorov's equation for nonlinear system (11) driven by non-Gaussian white noise

$\frac{\partial W}{\partial t}=-\frac{\partial[f(x, t) W]}{\partial x}+\int_{-\infty}^{\infty} \frac{\rho(z)}{z^{2}}\left[\exp \left\{-z \frac{\partial}{\partial x} g(x, t)\right\}-1+\sin z \frac{\partial}{\partial x} g(x, t)\right] d z W$, 
which is the main result of our paper. By series expansion of the exponential operator in Eq. (15), we have

$$
\frac{\partial W}{\partial t}=-\frac{\partial}{\partial x}[f(x, t) W]+\sum_{n=1}^{\infty} \frac{(-1)^{n} A_{n}}{n !}\left[\frac{\partial}{\partial x} g(x, t)\right]^{n} W,
$$

where

$$
A_{1}=\int_{-\infty}^{\infty} \frac{z-\sin z}{z^{2}} \rho(z) d z, \quad A_{n}=\int_{-\infty}^{\infty} z^{n-2} \rho(z) d z, \quad n \geq 2 .
$$

The equation (16) is valid if all integrals in Eq. (17) converge. It coincides with the result obtained in Ref. [10] for Poissonian white noise $\xi(t)$, if we interpret the coefficients $A_{n}$ as the moments of random jumps of $x(t)$. Moreover, this equation can be recast in the Kramers-Moyal form

$$
\frac{\partial W}{\partial t}=\sum_{n=1}^{\infty} \frac{(-1)^{n}}{n !} \frac{\partial^{n}}{\partial x^{n}}\left[K_{n}(x, t) W\right]
$$

where $K_{n}(x, t), n=1,2, \ldots$ are the kinetic coefficients.

\section{Particular cases of non-Gaussian noise sources}

Following Ref. [8] we analyze different kernel functions $\rho(x)$ to obtain particular cases of Kolmogorov's equation (15), related to different non-Gaussian noise sources.

(a) As a first simple case we consider a Gaussian white noise $\xi(t)$. The corresponding kernel function is $\rho(x)=2 D \delta(x)$. Therefore we obtain the ordinary Fokker-Planck equation

$$
\frac{\partial W}{\partial t}=-\frac{\partial}{\partial x}[f(x, t) W]+D \frac{\partial}{\partial x} g(x, t) \frac{\partial}{\partial x} g(x, t) W .
$$

(b) For Poisson kernel $\rho(x)=B \delta(x-q)$, Eq. (15) becomes

$$
\frac{\partial W}{\partial t}=-\frac{\partial}{\partial x}\left[f(x, t)+\frac{B g(x, t)(q-\sin q)}{q^{2}}\right] W+B \sum_{n=2}^{\infty} \frac{(-1)^{n} q^{n-2}}{n !}\left[\frac{\partial}{\partial x} g(x, t)\right]^{n} W .
$$

(c) When the increment $\eta_{T}$ of generalized Wiener process has the gamma distribution with exponent $\mu$, the kernel is $\rho(x)=\mu x e^{-a x}(x \geq 0)$. As a result, after integration we find from Eq. (15)

$$
\frac{\partial W}{\partial t}=-\frac{\partial}{\partial x}[f(x, t)-\mu g(x, t) \operatorname{arccot} a] W-\mu \ln \left[1+\frac{1}{a} \frac{\partial}{\partial x} g(x, t)\right] W .
$$

(d) For one-side $\alpha$-stable Lévy distributions the kernel function is $\rho(x)=\rho_{0} x^{1-\alpha}$ $(x \geq 0)$, and we get

$$
\begin{gathered}
\frac{\partial W}{\partial t}=-\frac{\partial}{\partial x}[f(x, t) W] \\
+\rho_{0} \int_{0}^{\infty} \frac{d z}{z^{\alpha+1}}\left[\exp \left\{-z \frac{\partial}{\partial x} g(x, t)\right\}-1+\frac{\partial}{\partial x} g(x, t) \sin z\right] W(x, t) .
\end{gathered}
$$


Generalized Wiener process and Kolmogorov's equation...

After rearrangements we obtain

$$
\frac{\partial W}{\partial t}=-\frac{\partial[f(x, t) W]}{\partial x}+\frac{\rho_{0}}{\alpha} \frac{\partial}{\partial x} g(x, t) \int_{0}^{\infty} \frac{d y}{y^{\alpha}}\left[\cos y-\exp \left\{-y \frac{\partial}{\partial x} g(x, t)\right\}\right] W .
$$

This equation is the fractional Fokker-Planck equation, and describes anomalous diffusion in the form of asymmetric Lévy flights.

(e) For additive driving noise $\xi(t), g(x, t)=1$ in Eq. (11), and the exponential operator in Eq. (15) reduces to the space shift operator. As a result, we find

$$
\frac{\partial W}{\partial t}=-\frac{\partial}{\partial x}[f(x, t) W]+\int_{-\infty}^{\infty} \frac{\rho(z)}{z^{2}}\left[W(x-z, t)-W(x, t)+\sin z \frac{\partial W(x, t)}{\partial x}\right] d z .
$$

Equation (23) is similar to the Kolmogorov-Feller equation for purely discontinuous Markovian processes $[4,11]$

$$
\frac{\partial W}{\partial t}=\nu \int_{-\infty}^{\infty} Q(x-z) W(z, t) d z-\nu W(x, t),
$$

where $Q(x)$ is probability density of jumps step, and $\nu$ is the constant mean rate of jumps. Putting $f(x, t)=0$ and comparing Eq. (23) with Eq. (24) we obtain the kernel function for this case

$$
\rho(x)=\nu x^{2} Q(x) .
$$

For non-Gaussian driving force $\xi(t)$, with symmetric $\alpha$-stable Lévy distribution, the kernel function is $\rho(x)=D|x|^{1-\alpha}$. As a result, Eq. (23) takes the following form

$$
\frac{\partial W}{\partial t}=-\frac{\partial}{\partial x}[f(x, t) W]+D \int_{-\infty}^{\infty} \frac{W(z, t)-W(x, t)}{|x-z|^{\alpha+1}} d z
$$

and describes the anomalous diffusion in form of symmetric Lévy flights.

(f) Finally, for multiplicative noise $\xi(t)$ with $g(x, t)=x$, the operator in Eq. (15) reduces to a scaling operator, and the generalized Kolmogorov's equation becomes

$$
\frac{\partial W}{\partial t}=-\frac{\partial}{\partial x}[f(x, t)-K x] W+\int_{-\infty}^{\infty} \frac{\rho(z)}{z^{2}}\left[W\left(e^{-z} x, t\right)-W(x, t)\right] d z,
$$

where

$$
K=\int_{-\infty}^{\infty} \frac{\rho(z) \sin z}{z^{2}} d z .
$$

\section{Simple examples of superdiffusion}

Now we derive the stationary probability distribution $W_{\infty}(x)$ for some simple cases of anomalous diffusion in a fixed potential $U(x)$ and in overdamped regime (see also $[6,12,13])$. The Langevin equation for the particle displacement $x(t)$ reads

$$
\dot{x}=-U^{\prime}(x)+\xi(t),
$$

where $\xi(t)$ is a symmetric $\alpha$-stable Lévy process. The corresponding Kolmogorov's equation is Eq. (26), with $f(x, t)=-U^{\prime}(x)$. The stationary probability distribution 
$W_{\infty}(x)$ can be found from the following equation

$$
\frac{d}{d x}\left[U^{\prime}(x) W_{\infty}\right]+D \int_{-\infty}^{\infty} \frac{W_{\infty}(z)-W_{\infty}(x)}{|x-z|^{\alpha+1}} d z=0
$$

a) For uniform potential well with two reflecting boundaries at $x= \pm L$ we get, from Eq. (29), the uniform stationary probability density

$$
W_{\infty}(x)=\frac{1}{2 L}
$$

as for ordinary Brownian diffusion.

b) For linear system with parabolic potential $U(x)=\beta x^{2} / 2$, by Fourier transform of Eq. (29), we obtain the asymptotic characteristic function of the process $x(t)$ as $[6]$

$$
\theta_{\infty}(k)=\exp \left\{-\frac{2 \Gamma(-\alpha) \cos (\pi \alpha / 2)}{\alpha \beta}|k|^{\alpha}\right\}
$$

c) For symmetric potential $U(x)=\gamma|x|$, after Fourier transform of Eq. (29) we find

$$
\gamma k G(k)+2 D|k|^{\alpha} \Gamma(-\alpha) F(k) \cos \frac{\pi \alpha}{2}=0,
$$

where

$$
F(k)=\int_{0}^{\infty} W_{\infty}(x) \cos k x d x, \quad G(k)=\int_{0}^{\infty} W_{\infty}(x) \sin k x d x
$$

Functions $F(k)$ and $G(k)$ are connected by Hilbert formula

$$
G(k)=\frac{1}{\pi} v \cdot p \cdot \int_{0}^{\infty} \frac{F(q)}{k-q} d q
$$

where v.p. means the principal value of integral. After substitution of Eq. (32) in Eq. (31) we obtain finally the following integral equation for cosine Fourier transform of stationary probability density

$$
v \cdot p \cdot \int_{0}^{\infty} \frac{F(q)}{k-q} d q+\frac{2 \pi D}{\gamma k}|k|^{\alpha} \Gamma(-\alpha) F(k) \cos \frac{\pi \alpha}{2}=0 .
$$

d) For quartic potential $U(x)=\beta x^{4} / 4$, from Eq. (29) we obtain the following differential equation for stationary characteristic function

$$
\beta k \frac{d^{3} \theta_{\infty}(k)}{d k^{3}}-2 D|k|^{\alpha} \Gamma(-\alpha) \theta_{\infty}(k) \cos \frac{\pi \alpha}{2}=0 .
$$

Equations (33) and (34) can be solved numerically. 
Generalized Wiener process and Kolmogorov's equation...

\section{Conclusions}

Starting from the generalized Wiener process, whose increments have the probability distribution belonging to the class of infinitely divisible distributions, we obtained the new correlation formula for stochastic functionals. This allows us to derive the Kolmogorov's equation (15) for diffusion, induced by non-Gaussian white noise source, directly from Langevin equation. The Fokker-Planck equation for ordinary Brownian motion, the Kolmogorov-Feller equation for purely discontinuous Markovian processes and the fractional Fokker-Planck equation for anomalous diffusion are obtained as particular cases of the Kolmogorov's equation (15). This equation can be used for determining the stationary probability distributions, probability distributions of first-passage times and other statistical characteristics of nonlinear dynamical systems.

\section{Acknowledgements}

This work has been supported by INTAS Grant 2001-0450, MIUR, INFM, by Russian Foundation for Basic Research (project 02-02-17517), by Federal Program "Scientific Schools of Russia" (project 1729.2003.2), and by Scientific Program "Universities of Russia" (project 01.01.020).

\section{References}

[1] S. M. Bezrukov and I. Vodyanoy, Noise-induced enhancement of signal transduction across voltage-dependent ion channels, Nature, 378 (1995), 362-364.

[2] P. Castiglione, A. Mazzino, P. Muratore-Ginanneschi, and A. Vulpiani, On strong anomalous diffusion, Physica D, 134 (1999), 75-93.

[3] R. Metzler and J. Klafter, The random walk's guide to anomalous diffusion: a fractional dynamics approach, Phys. Rep., 339 (2000), 1-77.

[4] A. I. Saichev and G. M. Zaslavsky, Fractional kinetic equations: solutions and applications, Chaos, 7 (1997), 753-764.

[5] B. J. West, P. Grigolini, R. Metzler, and T. F. Nonnenmacher, Fractional diffusion and Lévy stable processes, Phys. Rev. E, 55 (1997), 99-106.

[6] S. Jespersen, R. Metzler, and H. C. Fogedby, Lévy flights in external force fields: Langevin and fractional Fokker-Planck equations and their solutions, Phys. Rev. E, 59 (1999), 2736-2745.

[7] V. V. Yanovsky, A. V. Chechkin, D. Schertzer, and A. V. Tur, Lévy anomalous diffusion and fractional Fokker-Planck equation, Physica A, 282 (2000), 13-34.

[8] W. Feller, An Introduction to Probability Theory and its Applications, Vol. 2, John Wiley \& Sons, Inc., New York (1971).

[9] V. I. Klyatskin, To statistical theory of light reflection in randomly inhomogeneous medium, Sov. Phys. JETP, 38 (1974), 27-34.

[10] P. Hänggi, Correlation Functions and Master Equations of Generalized (NonMarkovian) Langevin Equations, Z. Physik B, 31 (1978), 407-416.

[11] A. Kamińska and T. Srokowski, Simple jumping process with memory: Transport equation and diffusion, Phys. Rev. E, 69 (2004), 062103-1 - 062103-4.

[12] A. V. Chechkin, V. Yu. Gonchar, J. Klafter, R. Metzler, and L. V. Tanatarov, Lévy Flights in a Steep Potential Well, J. Stat. Phys., 115 (2004), 1505 - 1535.

[13] Y. P. Kalmykov, W. T. Coffey, and S. V. Titov, Bimodal approximation for anomalous diffusion in a potential, Phys. Rev. E, 69 (2004), 021105-1 - 021105-7. 